\title{
General Method for Solving the Split Common Fixed Point Problem
}

\author{
Andrzej Cegielski
}

Received: 10 January 2014 / Accepted: 6 October 2014 / Published online: 21 October 2014

(C) The Author(s) 2014. This article is published with open access at Springerlink.com

\begin{abstract}
The split common fixed point problem (also called the multiple-sets split feasibility problem) is to find a common fixed point of a finite family of operators in one real Hilbert space, whose image under a bounded linear transformation is a common fixed point of another family of operators in the image space. In the literature one can find many methods for solving this problem as well as for its special case, called the split feasibility problem. We propose a general method for solving both problems. The method is based on a block-iterative procedure, in which we apply quasi-nonexpansive operators satisfying the demi-closedness principle and having a common fixed point. We prove the weak convergence of sequences generated by this method and show that the convergence for methods known from the literature follows from our general result.
\end{abstract}

Keywords Split feasibility problem $\cdot$ Split common fixed point problem .

Quasi-nonexpansive operators · Block-iterative procedure - Demi-closedness principle

Mathematics Subject Classification $\quad 47 \mathrm{~J} 25 \cdot 47 \mathrm{~N} 10 \cdot 65 \mathrm{~J} 15 \cdot 90 \mathrm{C} 25$

\section{Introduction}

In 1994 Censor and Elfving [1] introduced a notion of the split feasibility problem (SFP), which is to find an element of a closed convex subset of the Euclidean space

Communicated by Alfredo N. Iusem.

A. Cegielski $(\varangle)$

Faculty of Mathematics, Computer Science and Econometrics, University of Zielona

Góra, ul. Szafrana 4a, 65-516 Zielona Gora, Poland

e-mail: a.cegielski@wmie.uz.zgora.pl 
whose image under a linear operator is an element of another closed convex subset of a Euclidean space. Byrne [2] proposed a $C Q$-method for solving this problem and proved the convergence of sequences generated by this method to a solution. Qu and Xiu [3,4] studied the $C Q$-method in which the metric projections are replaced by subgradient projections, and proved the convergence of the method in the consistent case. Xu [5] extended the method of Qu and Xiu to Hilbert spaces and proved the weak convergence. Some versions of the method with an application of quasi-nonexpansive operators satisfying the demi-closedness principle were studied by Moudafi $[6,7]$.

The split feasibility problem is a special case of the split common fixed point problem (SCFPP), introduced by Censor and Segal [8]. The problem is to find a common fixed point of a finite family of operators defined on a real Hilbert space, whose image under a bounded linear transformation is a common fixed point of another family of operators defined on a real Hilbert space. The problem is also called the multiple-sets split feasibility problem (MSSFP) [9]. The problem was studied in [813], where various methods were proposed for solving it.

In this paper we study the behavior of a general method for solving the SCFPP; we prove the weak convergence of sequences generated by this method and show that the convergence of several known methods follows from the results presented in the paper.

The paper is organized as follows. In Sect. 2 we recall some facts regarding quasinonexpansive operators, which we apply in this paper. In Sect. 3 we present various methods known from the literature for solving the split common fixed point problem. In Sect. 4 we show that the class of strongly quasi-nonexpansive operators having a common fixed point and satisfying the demi-closedness principle is closed under convex combination and composition. The main result of the paper is contained in Sect. 5 , where we show in particular that a block-iterative $C Q$-type method with intermittent control in which the metric projections are replaced by strongly quasi-nonexpansive operators satisfying the demi-closedness principle generates sequences converging to a solution of a consistent SCFPP. In Sect. 6 we give several examples, where we show that the weak convergence of methods known from the literature follows from the results presented in Sect. 5.

\section{Preliminaries}

Let $\mathcal{H}$ be a real Hilbert space equipped with an inner product $\langle\cdot, \cdot\rangle$ and with the corresponding norm $\|\cdot\|$. In this Section we recall some definitions and facts which we apply later. We say that an operator $S: \mathcal{H} \rightarrow \mathcal{H}$ having a fixed point is $\alpha$-strongly quasi-nonexpansive ( $\alpha$-SQNE), where $\alpha \geq 0$, iff for all $x \in \mathcal{H}$ and all $z \in \operatorname{Fix} S$ it holds

$$
\|S x-z\|^{2} \leq\|x-z\|^{2}-\alpha\|S x-x\|^{2} .
$$

If $\alpha>0$, then we call an $\alpha$-SQNE operator strongly quasi-nonexpansive. A 0 -SQNE operator is called quasi-nonexpansive. An extended collection of properties of SQNE operators can be found, e.g., in [14, Chap. 2]. We say that $S$ is a cutter iff

$$
\langle x-S x, z-S x\rangle \leq 0
$$


for all $x \in \mathcal{H}$ and all $z \in$ Fix $S$. An operator $S$ is a cutter if and only if it is 1-SQNE. In the literature a cutter is also named a directed operator [8], a $\mathcal{T}$-class operator [15], a firmly quasi-nonexpansive operator [16], or a separating operator [17]. The name cutter was introduced in [18]. Denote by $S_{\lambda}:=\mathrm{Id}+\lambda(S-\mathrm{Id})$ a $\lambda$-relaxation of an operator $S$, where $\lambda \geq 0$.

Now we recall some properties of SQNE operators. An operator $U$ is $\alpha$-SQNE, where $\alpha \geq 0$, if and only if $U$ is a $\lambda$-relaxation of a cutter $S$ (also called a $\lambda$-relaxed cutter), where $\lambda \in] 0,2]$ and $\alpha=\frac{2-\lambda}{\lambda}$ (or, equivalently, $\lambda=\frac{2}{1+\alpha}$ ) [14, Theorem 2.1.39]. In particular, $U$ is QNE if and only if $U=2 S-$ Id for a cutter $S$ [15, Proposition 2.3]. The latter can also be expressed as follows: $U$ is QNE if and only if $S:=\frac{1}{2}(U+\mathrm{Id})$ is a cutter [14, Corollary 2.1.33(ii)]. Moreover, $U$ is a $\lambda$-relaxed cutter, where $\lambda \in] 0,2]$ (or, equivalently, $\alpha$-SQNE, where $\left.\alpha=\frac{2-\lambda}{\lambda} \in\right] 0,+\infty[$ ) if and only if

$$
\lambda\langle U x-x, z-x\rangle \geq\|U x-x\|^{2}
$$

for all $x \in \mathcal{H}$ and all $z \in$ Fix $U$ [14, Remark 2.1.31]. The above properties lead to the following: An operator $U$ is $\alpha$-SQNE, where $\alpha \geq 0$, if and only if $U$ is a $\lambda$-relaxation of a QNE operator, where $\lambda \in] 0,1]$ and $\alpha=\frac{1-\lambda}{\lambda}$.

Example 2.1 (metric projection) Let $C \subseteq \mathcal{H}$. A point $y \in C$ satisfying $\|y-x\| \leq$ $\|z-x\|$ for all $z \in C$ is called a metric projection of $x$ onto $C$. It is well known that if $C$ is closed convex, then for any $x \in \mathcal{H}$, the metric projection $P_{C} x$ exists and is defined uniquely. Moreover, $P_{C}$ is a cutter (see, e.g., [14, Theorem 2.2.21]).

Example 2.2 (subgradient projection) Let $f: \mathcal{H} \rightarrow \mathbb{R}$ be a continuous convex function with $S(f, 0):=\{x \in \mathcal{H}: f(x) \leq 0\} \neq \emptyset$. The subset

$$
\partial f(x):=\{g \in \mathcal{H}:\langle g, y-x\rangle \leq f(y)-f(x) \text { for all } y \in \mathcal{H}\}
$$

is called a subdifferential of $f$ at $x \in \mathcal{H}$, and its elements are called subgradients of $f$ at $x$. A subgradient projection $P_{f}: \mathcal{H} \rightarrow \mathcal{H}$ is defined as follows:

$$
P_{f}(x):= \begin{cases}x-\frac{f(x)}{\left\|g_{f}(x)\right\|^{2}} g_{f}(x), & \text { if } f(x)>0 \\ x, & \text { otherwise }\end{cases}
$$

where $g_{f}(x) \in \partial f(x)$ is a fixed subgradient, $x \in \mathcal{H}$. Note that for any $x \in \mathcal{H}$ the subgradient projection $P_{f}(x)$ depends on a selection $g_{f}(x) \in \partial f(x)$. Nevertheless, the properties of $P_{f}$ do not depend on these selections. We have Fix $P_{f}=S(f, 0)$, and $P_{f}$ is a cutter [14, Lemma 4.2.5 and Corollary 4.2.6]. For any $\left.\left.\lambda \in\right] 0,2\right]$, the relaxation $P_{f, \lambda}$ of a subgradient projection $P_{f}$ is $\alpha$-SQNE, where $\alpha=\frac{2-\lambda}{\lambda}$. In particular, $P_{f, 2}:=$ $2 P_{f}-$ Id is QNE. If we set $f(x)=d(x, C):=\inf _{y \in C}\|y-x\|$, where $C \subseteq \mathcal{H}$ is a closed convex subset, then $f$ is convex and differentiable for all $x \notin C$ and $g_{f}(x)=\frac{x-P_{C} x}{\left\|x-P_{C}\right\|}\left[14\right.$, Lemma 2.2.28]. Therefore, $P_{d(\cdot, C)}=P_{C}$, i.e., the metric projection is a special case of a subgradient projection. 
An important role in fixed point problems, especially in methods for solving SCFPPs, is played by operators satisfying the demi-closedness (DC) principle, i.e., operators $S: \mathcal{H} \rightarrow \mathcal{H}$ having the following property:

$$
\left(x^{k} \rightarrow x \text { and }\left\|S x^{k}-x^{k}\right\| \rightarrow 0\right) \Longrightarrow x \in \operatorname{Fix} S \text {. }
$$

We also say that $S-$ Id is demi-closed at 0 . Opial proved that nonexpansive operators satisfy the DC principle [19, Lemma 2]. The same property applies to subgradient projections for convex functions which are Lipschitz continuous on bounded subsets (see [14, Theorem 4.2.7] ). See [20] and [18] for related results.

\section{Split Common Fixed Point Problem and Solution Methods}

Let $\mathcal{H}, \mathcal{H}_{1}$, and $\mathcal{H}_{2}$ be real Hilbert spaces and $A: \mathcal{H}_{1} \rightarrow \mathcal{H}_{2}$ be a bounded linear operator with $\|A\|>0$. Further, let $U_{i}: \mathcal{H}_{1} \rightarrow \mathcal{H}_{1}, i \in I:=\{1,2, \ldots, p\}$ and $T_{j}: \mathcal{H}_{2} \rightarrow \mathcal{H}_{2}, j \in J:=\{1,2, \ldots, r\}$, be quasi-nonexpansive operators with $C:=\bigcap_{i \in I} \operatorname{Fix} U_{i}$ and $Q:=\bigcap_{j \in J} \operatorname{Fix} T_{j}$. The split common fixed point problem (SCFPP) is to

$$
\text { find } x^{*} \in \bigcap_{i \in I} \operatorname{Fix} U_{i} \text { with } A x^{*} \in \bigcap_{j \in J} \operatorname{Fix} T_{j} \text {. }
$$

The SCFPP in Euclidean spaces was introduced by Censor and Segal [8]. The problem is also called the multiple-sets split feasibility problem (MSSFP) [9], especially in the case if $C_{i}:=\operatorname{Fix} U_{i}$ and $Q_{j}:=$ Fix $T_{j}$ have the forms which allow us to calculate the metric projections $P_{C_{i}}$ onto $C_{i}$ and $P_{Q_{j}}$ onto $Q_{j}, i \in I, j \in J$. Denote $C:=\bigcap_{i \in I} C_{i}, Q:=\bigcap_{j \in J} Q_{j}$ and $F:=\{x \in C: A x \in Q\}=C \cap A^{-1}(Q)$. The MSSFP can be considered even if $F=\emptyset$ by introducing a proximity function $f: \mathcal{H}_{1} \rightarrow \mathbb{R}$. In this case one seeks a minimizer of $f$. Censor et al. [9] proposed the following proximity function:

$$
f(x):=\frac{1}{2} \sum_{i=1}^{p} \alpha_{i}\left\|P_{C_{i}} x-x\right\|^{2}+\frac{1}{2} \sum_{j=1}^{r} \beta_{j}\left\|P_{Q_{j}}(A x)-A x\right\|^{2},
$$

where $\alpha_{i}>0, i \in I$, and $\beta_{j}>0, j \in J$.

If $p=r=1$, then the MSSFP is reduced to the split feasibility problem (SFP):

$$
\text { find } x \in C \text { with } A x \in Q \text {. }
$$

The SFP was introduced by Censor and Elfving in [1]. Byrne [2] proposed the following method for solving the problem in $\mathbb{R}^{n}$ and called it the $C Q$-method:

$$
u^{k+1}=P_{C}\left(u^{k}+\frac{\lambda}{\|A\|^{2}} A^{*}\left(P_{Q}\left(A u^{k}\right)-A u^{k}\right)\right),
$$


where $u^{0} \in \mathbb{R}^{n}$ is arbitrary, $C \subseteq \mathbb{R}^{n}, Q \subseteq \mathbb{R}^{m}$ are closed convex subsets, $A$ is an $m \times n$ nonzero matrix, $\|A\|$ denotes its spectral norm and $\lambda \in] 0,2[$. Byrne proved the convergence of $x^{k}$ to a minimizer of a proximity function $f: C \rightarrow \mathbb{R}$ defined by

$$
f(x):=\frac{1}{2}\left\|P_{Q}(A x)-A x\right\|^{2},
$$

if such a minimizer exists. Note that the proximity function (7) with $p=r=1$ differs from (10), and in the case $F=\emptyset$ the minimizers of (7) and (10) may differ. Nevertheless, if $F \neq \emptyset$, then the subsets of minimizers of both proximity functions (7) with $p=r=1$ and (10) coincide, and any minimizer of these functions is a solution of (8). Moreover, if $F \neq \emptyset$, then the minimal value of both proximity functions is equal to 0 . If $Q$ is a solution set of a system of linear equations, then iteration (9) is known as the projected Landweber (PL) method [2]. This name we will also use in the general case. Yang [21] studied the SFP in $\mathbb{R}^{n}$ with $F \neq \emptyset$ and modified the PL method by replacing the metric projections $P_{C}$ and $P_{Q}$ in (9) by subgradient projections. More precisely, the method has the form

$$
u^{k+1}=P_{c}\left(u^{k}+\frac{\lambda}{\|A\|^{2}} A^{*}\left(P_{q}\left(A u^{k}\right)-A u^{k}\right)\right),
$$

where $u^{0} \in \mathbb{R}^{n}$ is arbitrary, $P_{c}$ and $P_{q}$ denote subgradient projections for $c: \mathbb{R}^{n} \rightarrow \mathbb{R}$ and $q: \mathbb{R}^{m} \rightarrow \mathbb{R}$ being convex functions, $C:=\left\{x \in \mathbb{R}^{n}: c(x) \leq 0\right\} \neq \emptyset$ and $Q:=\left\{y \in \mathbb{R}^{m}: q(y) \leq 0\right\} \neq \emptyset$. Qu and Xiu studied the convergence of the PL method (9) in $\mathbb{R}^{n}$, where the constant $\lambda$ was replaced by $\lambda_{k}$ which was calculated by the Armijo-like search rule [3, Theorem 3.1]. Qu and Xiu also studied method (9) in which the metric projections $P_{C}$ and $P_{Q}$ are replaced by subgradient projections $P_{c}$ and $P_{q}$. This study was continued in [4], where a method related to a reformulated problem was proposed. Cegielski [22] studied the convergence of an extrapolated PL method for a linear split feasibility problem in $\mathbb{R}^{n}$. Xu studied the SFP with $F \neq \varnothing$ and proved the weak convergence of sequences generated by method (11) in Hilbert spaces [5, Theorem 4.1]. Zhao and Yang [23] studied the PL method in Euclidean spaces, where the metric projections onto $C$ and $Q$ were replaced by metric projections onto subsets $C_{k}$ and $Q_{k}$ which converge in the Mosco sense to $C$ and $Q$, respectively, and proposed sufficient conditions by which the sequence $u^{k}$ converges to a solution. Moudafi [6] studied the SFP with $F \neq \emptyset$ in Hilbert spaces and proposed the following method:

$$
u^{k+1}=U_{\alpha_{k}}\left(u^{k}+\gamma A^{*}\left(T\left(A u^{k}\right)-A u^{k}\right)\right),
$$

where $u^{0} \in \mathcal{H}_{1}$ is arbitrary, $U: \mathcal{H}_{1} \rightarrow \mathcal{H}_{1}$ and $T: \mathcal{H}_{2} \rightarrow \mathcal{H}_{2}$ are demi-contractive operators with Fix $U=C \neq \emptyset$ and Fix $T=Q \neq \emptyset$, satisfying the demi-closedness principle, $\alpha_{k}$ and $\gamma$ are some positive parameters, and $U_{\alpha}:=\mathrm{Id}+\alpha(U-\mathrm{Id})$ denotes the $\alpha$-relaxation of $U$. The study was continued in [7], where $U$ and $T$ are supposed to be quasi-nonexpansive operators satisfying the demi-closedness principle, $0<\gamma<$ $1 /\|A\|^{2}, \alpha_{k} \in(\delta, 1-\delta)$ for some small $\delta>0$. Censor et al. [10] studied SCFPP in $\mathbb{R}^{n}$ with $F \neq \emptyset$ and proposed the following method: 


$$
u^{k+1}=u^{k}+\gamma\left(\sum_{i=1}^{p} \alpha_{i}\left(P_{c_{i}} u^{k}-u^{k}\right)+\sum_{j=1}^{r} \beta_{j} A^{*}\left(P_{q_{j}}\left(A u^{k}\right)-A u^{k}\right)\right),
$$

where $u^{0} \in \mathbb{R}^{n}$ is arbitrary, $\alpha_{i}>0, \beta_{j}>0, c_{i}: \mathbb{R}^{n} \rightarrow \mathbb{R}$ and $q_{j}: \mathbb{R}^{m} \rightarrow \mathbb{R}$ are convex functions, $C_{i}:=\left\{x \in \mathbb{R}^{n}: c_{i}(x) \leq 0\right\} \neq \emptyset, Q_{j}:=\left\{y \in \mathbb{R}^{m}: q_{j}(y) \leq 0\right\} \neq \emptyset$, $i \in I, j \in J$, and

$$
0<\gamma<2 /\left(\sum_{i=1}^{p} \alpha_{i}+\|A\|^{2} \sum_{j=1}^{r} \beta_{j}\right)
$$

This study was continued by Censor and Segal in [8], where the subgradient projections $P_{c_{i}}$ and $P_{q_{j}}$ in (13) were replaced by directed operators $U_{i}$ and $T_{j}, i \in I, j \in J$, satisfying the closedness principle. The iteration proposed by Censor and Segal has the form

$$
u^{k+1}=u^{k}+\gamma\left(\sum_{i=1}^{p} \alpha_{i}\left(U_{i} u^{k}-u^{k}\right)+\sum_{j=1}^{r} \beta_{j} A^{*}\left(T_{j}\left(A u^{k}\right)-A u^{k}\right)\right) .
$$

Some further algorithms for solving the SCFPP with $F \neq \emptyset$, defined in Hilbert spaces were proposed by $\mathrm{Xu}$ in [12, Sect. 3.2]. Denote

$$
P:=\sum_{i=1}^{p} \alpha_{i} P_{C_{i}}
$$

where $\alpha_{i}>0, i \in I, \sum_{i=1}^{p} \alpha_{i}=1$ and

$$
S:=\mathrm{Id}+\gamma \sum_{j=1}^{r} \beta_{j}\left(A^{*}\left(P_{Q_{j}}-\mathrm{Id}\right) A\right)
$$

where $\beta_{j}>0, j \in J$, and $0<\gamma<2 /\left(\|A\|^{2} \sum_{j=1}^{r} \beta_{j}\right)$ and Id denotes the identity operator. $\mathrm{Xu}$ proposed three algorithms presented below and proved the weak convergence to a solution for sequences generated by these algorithms:

$$
u^{k+1}=P_{C_{p}} S P_{C_{p-1}} S \ldots P_{C_{1}} S u^{k},
$$

[12, Theorem 3.3],

$$
u^{k+1}=P S u^{k}
$$

[12, Theorem 3.4] and

$$
u^{k+1}=P_{C_{i_{k}}} S u^{k},
$$

where $u^{0} \in \mathcal{H}_{1}$ is arbitrary, $i_{k}=k(\bmod p)+1$ [12, Theorem 3.5]. SCFPP (6) with $p=r$ and $F \neq \varnothing$ defined in Hilbert spaces was studied by Wang and $\mathrm{Xu}$ in [13, Lemma 3.1], where the following method was proposed for solving this problem: 


$$
u^{k+1}=U_{i_{k}}\left(u^{k}+\frac{\lambda}{\|A\|^{2}} A^{*}\left(T_{i_{k}} A u^{k}-A u^{k}\right)\right),
$$

where $u^{0} \in \mathcal{H}_{1}$ is arbitrary, $\left.\lambda \in\right] 0,2\left[, U_{i}, T_{i}\right.$ are $\mathcal{T}$-class operators satisfying the demi-closedness principle, $i \in I$, and $i_{k}$ is a cyclic control, i.e., $i_{k}=k(\bmod p)+1$. Censor et al. [9] introduced the following constrained multiple-sets split feasibility problem: find $x \in \Omega$ such that $x \in C:=\bigcap_{i \in I} C_{i}$ with $A x \in Q:=\bigcap_{j \in J} Q_{j}$, where $\Omega, C_{i} \subseteq \mathbb{R}^{n}, Q_{j} \subseteq \mathbb{R}^{m}$ are closed convex subsets, $i \in I, j \in J$, and proposed the following method for solving the problem:

$$
u^{k+1}=P_{\Omega}\left(u^{k}+\gamma\left(\sum_{i=1}^{p} \alpha_{i}\left(P_{C_{i}} u^{k}-u^{k}\right)+\sum_{j=1}^{r} \beta_{j} A^{*}\left(P_{Q_{j}} A u^{k}-A u^{k}\right)\right)\right),
$$

where $u^{0} \in \mathbb{R}^{n}$ is arbitrary, $\alpha_{i}>0, \beta_{j}>0, i \in I, j \in J$, and $\gamma$ satisfies (14). Censor et al. proved the convergence of $u^{k}$ to a minimizer of the proximity function $f: \Omega \rightarrow \mathbb{R}$ defined by (7) [9, Theorem 3]. Masad and Reich [11] generalized this result and proved the weak convergence in a Hilbert space. In Sect. 5 we suppose that $F \neq \varnothing$ and prove that the weak convergence of sequences generated by methods (9), (11) (12), (13), (15), (16), (17), (18), and (19) presented above follows from a general result.

\section{Properties of Quasi-nonexpansive Operators Satisfying the Demi-closedness Principle}

In this section we present some properties of QNE operators satisfying the DC principle, which we will apply in Sect. 5. The following Lemma is a slight generalization of [13, Lemma 3.1 and Theorem 3.3].

Lemma 4.1 Let $A: \mathcal{H}_{1} \rightarrow \mathcal{H}_{2}$ be a bounded linear operator with $\|A\|>0$ and $T: \mathcal{H}_{2} \rightarrow \mathcal{H}_{2}$ be an operator satisfying $T A w=A w$ for some $w \in \mathcal{H}_{1}$. Further, let $V:=\mathrm{Id}+\frac{1}{\|A\|^{2}} A^{*}(T-I d) A$. If $T$ is $\alpha$-SQNE, where $\alpha \geq 0$, then

(i) Fix $V=A^{-1}($ Fix $T)$ and

(ii) $V$ is $\alpha-S Q N E$.

If, moreover, $T$ satisfies the DC principle, then $V$ also satisfies the DC principle.

Proof As mentioned before, $T$ is $\alpha$-SQNE, where $\alpha \geq 0$, if and only if

$$
\lambda\langle T u-u, y-u\rangle \geq\|T u-u\|^{2}
$$

for all $u \in \mathcal{H}_{2}$ and all $y \in \operatorname{Fix} T$, where $\left.\left.\lambda=2 /(\alpha+1) \in\right] 0,2\right]$. Note that $z \in$ $A^{-1}($ Fix $T)$ if and only if $A z \in$ Fix $T$.

(i) $\supseteq$ Let $A z \in \operatorname{Fix} T$. Then $V z=z+\left(\frac{1}{\|A\|^{2}} A^{*}(T A z-A z)=z\right.$ 
$\subseteq$ Let $z \in$ Fix $V$. Then, of course, $A^{*}(T A z-A z)=0$. Let $w \in \mathcal{H}_{1}$ be such that $A w \in$ Fix $T$. We have

$$
\begin{aligned}
& \|T A z-A z\|^{2} \leq \lambda\langle T A z-A z, A w-A z\rangle=\lambda\left\langle A^{*}(T A z-A z), w-z\right\rangle=0, \\
& \text { i.e., } A z \in \text { Fix } T \text {. }
\end{aligned}
$$

(ii) Let $z \in \operatorname{Fix} V$. Because $A z \in \operatorname{Fix} T$, inequality (21) and equality $\left\|A^{*}\right\|=\|A\|$ yield

$$
\begin{aligned}
\lambda\langle V x-x, z-x\rangle & =\lambda\left\langle\frac{1}{\|A\|^{2}} A^{*}(T A x-A x), z-x\right\rangle \\
& =\frac{\lambda}{\|A\|^{2}}\langle(T A x-A x), A z-A x\rangle \\
& \geq \frac{1}{\|A\|^{2}}\|T A x-A x\|^{2}=\frac{\left\|A^{*}\right\|^{2}}{\|A\|^{4}}\|T A x-A x\|^{2} \\
& \geq \frac{1}{\|A\|^{4}}\left\|A^{*}(T A x-A x)\right\|^{2}=\left\|\frac{1}{\|A\|^{2}} A^{*}(T A x-A x)\right\|^{2} \\
& =\|V x-x\|^{2},
\end{aligned}
$$

i.e., $V$ is a $\lambda$-relaxed cutter, or, equivalently, an $\alpha$-SQNE operator.

Now suppose that $T$ satisfies the demi-closedness principle, i.e., $y^{k} \rightarrow y$ together with $\left\|T y^{k}-y^{k}\right\| \rightarrow 0$ imply $y \in$ Fix $T$. We prove that $V$ also satisfies the demiclosedness principle. Let $x^{k} \rightarrow x$ and $\left\|V x^{k}-x^{k}\right\| \rightarrow 0$. Clearly, for any $u \in \mathcal{H}_{2}$

$$
\lim _{k}\left\langle A x^{k}-A x, u\right\rangle=\lim _{k}\left\langle x^{k}-x, A^{*} u\right\rangle=0,
$$

i.e., $A x^{k} \rightarrow A x$. Choose an arbitrary $z \in$ Fix $V$. By (i), $A z \in$ Fix $T$. By (21), by the boundedness of $x^{k}$ and by the Cauchy-Schwarz inequality, we have

$$
\begin{aligned}
\left\|T A x^{k}-A x^{k}\right\|^{2} & \leq \lambda\left\langle T A x^{k}-A x^{k}, A z-A x^{k}\right\rangle \\
& =\lambda\|A\|^{2}\left\langle\frac{1}{\|A\|^{2}} A^{*}\left(T A x^{k}-A x^{k}\right), z-x^{k}\right\rangle \\
& \leq \lambda\|A\|^{2}\left\|V x^{k}-x^{k}\right\| \cdot\left\|z-x^{k}\right\| \rightarrow 0 \text { as } k \rightarrow \infty .
\end{aligned}
$$

Consequently, $\lim _{k}\left\|T\left(A x^{k}\right)-A x^{k}\right\|=0$. This, together with $A x^{k} \rightarrow A x$, gives $A x \in$ Fix $T$, i.e., $x \in A^{-1}(\operatorname{Fix} T)=$ Fix $V$, and the proof is completed.

Remark 4.1 If we set $\alpha=1$ in Lemma 4.1, then $T$ and $V$ are cutters, and we obtain the result of Wang and Xu [13, Lemma 3.1 and Theorem 3.3]. 
Denote $L:=\{1,2, \ldots, m\}$ and

$$
\Delta_{m}:=\left\{w=\left(\omega_{1}, \omega_{2}, \ldots, \omega_{m}\right) \in \mathbb{R}^{m}: \omega_{i} \geq 0 \text { and } \sum_{i=1}^{m} \omega_{i}=1\right\}
$$

Theorem 4.1 Let $S_{i}: \mathcal{H} \rightarrow \mathcal{H}$ be $\rho_{i}-S Q N E$ operators satisfying the DC principle, $i \in L, \bigcap_{i \in L}$ Fix $S_{i} \neq \emptyset$ and let $S=\sum_{i=1}^{m} \omega_{i} S_{i}$, where $w \in \Delta_{m}$. Then $S$ is a $\rho$-SQNE operator, with $\rho:=\sum_{i=1}^{m}\left(\frac{\omega_{i}}{\rho_{i}+1}\right)^{-1}-1$ and $S$ satisfies the DC principle.

Proof The fact that $S$ is $\rho$-SQNE follows from [14, Theorem 2.1.50]. Now we prove that $S$ satisfies the DC principle. Let $z \in \bigcap_{i \in L}$ Fix $S_{i}, x^{k} \rightarrow x^{*} \in \mathcal{H}$ and $\left\|S x^{k}-x^{k}\right\| \rightarrow 0$. Clearly, $x^{k}$ is bounded as a weakly convergent sequence. Therefore, there is $R>0$ such that $\left\|x^{k}-z\right\| \leq R$. By [24, Proposition 4.5], we have

$$
\frac{1}{2 R} \sum_{i=1}^{m} \omega_{i} \rho_{i}\left\|S_{i} x^{k}-x^{k}\right\|^{2} \leq\left\|S x^{k}-x^{k}\right\|
$$

consequently, $\left\|S_{i} x^{k}-x^{k}\right\| \rightarrow 0, i \in L$. The demi-closedness of $S_{i}-$ Id at 0 yields now $x^{*} \in \operatorname{Fix} S_{i}, i \in L$, i.e., $x^{*} \in \bigcap_{i \in L} \operatorname{Fix} S_{i}=\operatorname{Fix} S$ [25, Proposition 2.12(i)], and the proof is completed.

Theorem 4.2 Let $S_{i}: \mathcal{H} \rightarrow \mathcal{H}$ be $\rho_{i}$-SQNE operators satisfying the DC principle, $i \in L, \bigcap_{i \in L} \operatorname{Fix}_{i} \neq \varnothing$ and let $S=S_{m} S_{m-1} \ldots S_{1}$.Then $S$ is a $\rho$-SQNE operator, with $\rho=\left(\sum_{i=1}^{m} \rho_{i}^{-1}\right)^{-1}$, and $S$ satisfies the DC principle.

Proof The fact that $S$ is $\rho$-SQNE follows from [14, Theorem 2.1.48]. Now we prove that $S$ satisfies the DC principle. Without loss of generality we suppose that $m=2$. Let $z \in \operatorname{Fix} S_{1} \cap \operatorname{Fix} S_{2}, x^{k} \rightarrow x^{*} \in \mathcal{H}$ and $\left\|S x^{k}-x^{k}\right\| \rightarrow 0$. Clearly, $x^{k}$ is bounded as a weakly convergent sequence. Therefore, there is $R>0$ such that $\left\|x^{k}-z\right\| \leq R$. By [24, Proposition 4.6], we have

$$
\frac{1}{2 R}\left(\rho_{1}\left\|S_{1} x^{k}-x^{k}\right\|^{2}+\rho_{2}\left\|S_{2} S_{1} x^{k}-S_{1} x^{k}\right\|^{2}\right) \leq\left\|S x^{k}-x^{k}\right\|,
$$

where $\rho_{i}>0$ denotes the SQNE constant of $S_{i}, i=1,2$. Consequently, $\left\|S_{1} x^{k}-x^{k}\right\| \rightarrow 0$ and $\left\|S_{2} S_{1} x^{k}-S_{1} x^{k}\right\| \rightarrow 0$. The demi-closedness of $S_{1}-$ Id at 0 yields $x^{*} \in \operatorname{Fix} S_{1}$. Furthermore,

$$
y^{k}:=S_{1} x^{k}=x^{k}+\left(S_{1} x^{k}-x^{k}\right) \rightarrow x^{*} .
$$

The demi-closedness of $S_{2}-$ Id at 0 yields $x^{*} \in$ Fix $S_{2}$. Consequently, $x^{*} \in \operatorname{Fix} S_{1} \cap$ Fix $S_{2}=$ Fix $S$ [25, Proposition 2.10(i)], and the proof is completed.

Consider a method, which is slightly more general than the cyclic method of Wang an $\mathrm{Xu}$ (19). Suppose that $p=r, U_{i}, V_{i}, i \in I$, are cutters, relaxation parameters $\lambda, \mu \in] 0,2[$ and define the following iteration: 


$$
u^{k+1}=U_{i_{k}, \mu}\left(u^{k}+\frac{\lambda}{\|A\|^{2}} A^{*}\left(T_{i_{k}} A u^{k}-A u^{k}\right)\right),
$$

where $i_{k}$ is the cyclic control, i.e., $i_{k}=k(\bmod p)+1$. Applying notation $(28)$ we can write method (23) in the form

$$
u^{k+1}=U_{i_{k}, \mu} V_{i_{k}, \lambda} u^{k}
$$

Define

$$
U=U_{p, \mu} V_{p, \lambda} U_{p-1, \mu} V_{p-1, \lambda} \ldots U_{1, \mu} V_{1, \lambda}
$$

and consider the iteration

$$
y^{k+1}=U y^{k} .
$$

Note that $U$ is an SQNE operator satisfying the DC principle as a composition of SQNE operators satisfying the DC principle. Therefore, we easily obtain the following.

Proposition 4.1 For any starting point $y^{0} \in \mathcal{H}_{1}$ the sequence $y^{k}$ generated by (25) converges weakly to a point $y^{*} \in F$.

Proof Since an SQNE operator $U$ is asymptotically regular (see, e.g., [14, Theorem 3.4.3]), the proposition follows from [14, Theorem 3.6.2(i)].

The relationship $y^{k}=u^{p k}$ between the sequences $u^{k}$ and $y^{k}$ generated by (24) and (25) is clear. Consequently, $\left\{y^{k}\right\}_{k=0}^{\infty}$ is a subsequence of $\left\{u^{k}\right\}_{k=0}^{\infty}$. This observation together with Proposition 4.1 leads to the following result which is quite more general than [13, Theorem 3.2].

Corollary 4.1 For any starting point $u^{0} \in \mathcal{H}_{1}$ the sequence $u^{k}$ generated by (24) converges weakly to a point $u^{*} \in F$.

Proof Clearly, the sequence $\left\{u^{k}\right\}_{k=0}^{\infty}$ is Fejér monotone with respect to $F$ (i.e., for any $z \in F$ the sequence $\left\{\left\|u^{k}-z\right\|\right\}_{k=0}^{\infty}$ is decreasing), because the operators $U_{i, \mu}$ and $V_{i, \lambda}, i=1,2, \ldots, p$, are SQNE. It follows from Proposition 4.1 that the sequence $\left\{u^{p k}\right\}_{k=0}^{\infty}$ converges weakly to an element of $F$ as $k \rightarrow+\infty$. In a similar way one can prove that for any $i=1,2, \ldots, p-1$ the sequence $\left\{u^{p k+i}\right\}_{k=0}^{\infty}$ converges weakly to an element of $F$ as $k \rightarrow+\infty$. Therefore, all cluster points of the sequence $\left\{u^{k}\right\}_{k=0}^{\infty}$ belong to $F$ and the weak convergence of $u^{k}$ to an element of $F$ follows from [25, Theorem 2.16(ii)].

In the next section we will prove a result which is stronger than Corollary 4.1.

\section{Convergence Results}

In this section we study the behavior of the following method for solving the SCFPP:

$$
x^{k+1}=x^{k}+\gamma_{k}\left(\sum_{i=1}^{p} \alpha_{i}^{k}\left(U_{i} x^{k}-x^{k}\right)+\sum_{j=1}^{r} \beta_{j}^{k} A^{*}\left(T_{j} A x^{k}-A x^{k}\right)\right) \text {, }
$$


where $x^{0} \in \mathcal{H}$ is arbitrary, $\alpha_{i}^{k} \geq 0, i \in I, \beta_{j}^{k} \geq 0, j \in J$, and

$$
0<\gamma_{k}<1 /\left(\sum_{i=1}^{p} \alpha_{i}^{k}+\|A\|^{2} \sum_{j=1}^{r} \beta_{j}^{k}\right)
$$

$k \geq 0$. Since an operator $U$ is QNE if and only if $S:=\frac{1}{2}(U+\mathrm{Id})$ is a cutter, we can suppose equivalently that $U_{i}$ and $T_{j}, i \in I, j \in J$, are cutters in (26) and that

$$
0<\gamma_{k}<2 /\left(\sum_{i=1}^{p} \alpha_{i}^{k}+\|A\|^{2} \sum_{j=1}^{r} \beta_{j}^{k}\right)
$$

Define

$$
V_{j}=\mathrm{Id}+\left(\frac{1}{\|A\|^{2}} A^{*}\left(T_{j}-\mathrm{Id}\right) A\right),
$$

$j \in J$, where Id denotes the identity operator. Then we obtain the following form of (26):

$$
x^{k+1}=x^{k}+\lambda_{k}\left(\sum_{i=1}^{p} v_{i}^{k}\left(U_{i} x^{k}-x^{k}\right)+\sum_{j=1}^{r} \mu_{j}^{k}\left(V_{j} x^{k}-x^{k}\right)\right)
$$

where

$$
\begin{gathered}
\lambda_{k}=\gamma_{k}\left(\sum_{i=1}^{p} \alpha_{i}^{k}+\|A\|^{2} \sum_{j=1}^{r} \beta_{j}^{k}\right), \\
\nu_{i}^{k}=\alpha_{i}^{k} \gamma_{k} / \lambda_{k} \geq 0, i \in I, \\
\mu_{j}^{k}=\beta_{j}^{k}\|A\|^{2} \gamma_{k} / \lambda_{k} \geq 0, j \in J,
\end{gathered}
$$

and

$$
\sum_{i=1}^{p} v_{i}^{k}+\sum_{j=1}^{r} \mu_{j}^{k}=1
$$

Moreover, $\lambda_{k} \in$ ]0, 1 [ if we apply SQNE operators $U_{i}, V_{j}$ in (26) and in (29), and $\lambda_{k} \in$ ]0, 2[ if we apply cutter operators $U_{i}, V_{j}$ in (26) and in (29).

First we consider a special case of (29) in the form of the following block-iterative procedure:

$$
x^{k+1}=x^{k}+\mu_{k}\left(\sum_{i=1}^{m} \omega_{i}^{k} U_{i} x^{k}-x^{k}\right),
$$

where $x^{0} \in \mathcal{H}$ is arbitrary, $U_{i}: \mathcal{H} \rightarrow \mathcal{H}$ are QNE operators, $i \in L:=\{1,2, \ldots, m\}$, $\mu_{k} \in[\varepsilon, 1-\varepsilon]$ for some small $\varepsilon>0$ and $w^{k}=\left(\omega_{1}^{k}, \omega_{2}^{k}, \ldots, \omega_{m}^{k}\right) \in \Delta_{m}$. Denote $L_{k}:=\left\{i \in L: \omega_{i}^{k}>0\right\}, k \geq 0$.

Before we formulate a convergence theorem, we recall the notion of an intermittent control (cf. [25, Definition 3.18]). 
Definition 5.1 Let $L_{k} \subseteq L:=\{1,2, \ldots, m\}, k \geq 0$. We say that the sequence $\left\{L_{k}\right\}_{k=0}^{\infty}$ is $s$-intermittent, where $s \geq 1$, or intermittent, iff for any $k \geq 0$ it holds

$$
L_{k} \cup L_{k+1} \cup \ldots \cup L_{k+s-1}=L
$$

If $L_{k}=\left\{l_{k}\right\}$, for all $k \geq 0$, i.e., $L_{k}$ are 1-element subsets, then an $s$-intermittent sequence $\left\{l_{k}\right\}_{k=0}^{\infty}$ is called s-almost cyclic or almost cyclic. An $m$-almost cyclic sequence $\left\{l_{k}\right\}_{k=0}^{\infty}$ is called cyclic.

If we apply intermittent (almost cyclic, cyclic) sequences to a block-iterative procedure (34), then we say that the control in (34) is intermittent (almost cyclic, cyclic). If we apply a cyclic control $\left\{l_{k}\right\}_{k=0}^{\infty}$, then we can suppose without loss of generality that $l_{k}=k(\bmod m)+1$.

Theorem 5.1 Let $U_{i}: \mathcal{H} \rightarrow \mathcal{H}$ be $Q N E, i \in L, C:=\bigcap_{i \in L}$ Fix $U_{i} \neq \emptyset$ and $U_{i}$ satisfy the DC principle, $i \in L$. Let $\left\{x^{k}\right\}_{k=0}^{\infty}$ be generated by iteration (34) with $w^{k} \in \Delta_{m}$, where

$$
\omega_{i}^{k}>\delta>0 \text { for all } i \in L_{k}
$$

If $\left\{L_{k}\right\}_{k=0}^{\infty}$ is intermittent, then for arbitrary starting point $x^{0} \in \mathcal{H}$ the sequence $x^{k}$ converges weakly to a point $x^{*} \in \bigcap_{i \in L} F_{i x} U_{i}$.

Proof Obviously, Fix $T_{k} \supseteq C$. Iteration (34) can be written in the form $x^{k+1}=T_{k} x^{k}$, where $T_{k}:=\operatorname{Id}+\alpha_{k}\left(\sum_{i=1}^{m} \omega_{i}^{k} S_{i}-\mathrm{Id}\right), S_{i}:=\frac{1}{2}\left(U_{i}+\mathrm{Id}\right), i \in L$, are cutters and $\alpha_{k}=2 \mu_{k}$. Let $z \in F$. We have

$$
\left\|T_{l} x-z\right\|^{2} \leq\|x-z\|^{2}-\alpha_{l}\left(2-\alpha_{l}\right) \sum_{i=1}^{m} \omega_{i}^{l}\left\|S_{i} x-x\right\|^{2},
$$

$l=0$ (see [14, Theorem 4.8.2]). This together with the convexity of the function $\|\cdot\|^{2}$ yields

$$
\begin{aligned}
\left\|T_{l} x-z\right\|^{2} & \leq\|x-z\|^{2}-\mu_{l}\left(1-\mu_{l}\right) \sum_{i=1}^{m} \omega_{i}^{l}\left\|U_{i} x-x\right\|^{2} \\
& \leq\|x-z\|^{2}-\mu_{l}\left(1-\mu_{l}\right)\left\|\sum_{i=1}^{m} \omega_{i}^{l} U_{i} x-x\right\|^{2} \\
& =\|x-z\|^{2}-\frac{1-\mu_{l}}{\mu_{l}}\left\|T_{l} x-x\right\|^{2}
\end{aligned}
$$

for all $x \in \mathcal{H}_{1}, l \geq 0$, in particular, for $l=k$ and $x=x^{k}$. Therefore, $\left\|x^{k}-z\right\|$ is decreasing, and $\left\{x^{k}\right\}_{k=0}^{\infty}$ is bounded. Let $x^{*}$ be a weak cluster point of $\left\{x^{k}\right\}_{k=0}^{\infty}$ and let $\left\{x^{n_{k}}\right\}_{k=0}^{\infty} \subseteq\left\{x^{k}\right\}_{k=0}^{\infty}$ be a subsequence with

$$
x^{n_{k}} \rightarrow x^{*}
$$


Let $s \geq 1$ be such that $L_{k} \cup L_{k+1} \cup \ldots \cup L_{k+s-1}=L$ for all $k \geq 0$. If we set $l=n_{k}+s-1$ and $x=x^{n_{k}+s-1}$ in (35), then we obtain

$$
\begin{aligned}
\left\|x^{n_{k}+s}-z\right\|^{2}= & \left\|T_{n_{k}+s-1} x^{n_{k}+s-1}-z\right\|^{2} \\
\leq & \left\|x^{n_{k}+s-1}-z\right\|^{2}-\mu_{n_{k}+s-1}\left(1-\mu_{n_{k}+s-1}\right) \\
& \times \sum_{i=1}^{m} \omega_{i}^{n_{k}+s-1}\left\|U_{i} x^{n_{k}+s-1}-x^{n_{k}+s-1}\right\|^{2} \\
\leq & \left\|x^{n_{k}+s-1}-z\right\|^{2}-\frac{1-\mu_{n_{k}+s-1}}{\mu_{n_{k}+s-1}}\left\|T_{n_{k}+s-1} x^{n_{k}+s-1}-x^{n_{k}+s-1}\right\|^{2} .
\end{aligned}
$$

If we iterate the above inequalities $s$-times, we obtain

$$
\begin{aligned}
\left\|x^{n_{k}+s}-z\right\|^{2} & \leq\left\|x^{n_{k}}-z\right\|^{2}-\sum_{j=0}^{s-1} \mu_{n_{k}+j}\left(1-\mu_{n_{k}+j}\right) \sum_{i=1}^{m} \omega_{i}^{n_{k}+j}\left\|U_{i} x^{n_{k}+j}-x^{n_{k}+j}\right\|^{2} \\
& \leq\left\|x^{n_{k}}-z\right\|^{2}-\sum_{j=0}^{s-1} \frac{1-\mu_{n_{k}+j}}{\mu_{n_{k}+j}}\left\|T_{n_{k}+j} x^{n_{k}+j}-x^{n_{k}+j}\right\|^{2} \cdot
\end{aligned}
$$

Let $i \in L$ be arbitrary and let $r_{k} \in\{0,1, \ldots, s-1\}$ be such that $i \in L_{n_{k}+r_{k}}$. Because $\left\|x^{k}-z\right\|$ is decreasing, $\lim _{k}\left\|x^{n_{k}+s}-z\right\|=\lim _{k}\left\|x^{n_{k}}-z\right\|$. Furthermore, the inequalities $0<\varepsilon \leq \mu_{n_{k}+j} \leq 1-\varepsilon<1$ yield

$$
\lim _{k}\left\|x^{n_{k}+j+1}-x^{n_{k}+j}\right\|=\lim _{k}\left\|T_{n_{k}+j} x^{n_{k}+j}-x^{n_{k}+j}\right\|=0
$$

for all $j \in\{0,1, \ldots, s-1\}$ and

$$
\lim _{k}\left\|U_{i} x^{n_{k}+r_{k}}-x^{n_{k}+r_{k}}\right\|=0
$$

by $\omega_{i}^{n_{k}+r_{k}}>\delta>0$. Now (36) and (37) yield

$$
x^{n_{k}+r_{k}}=x^{n_{k}}+\sum_{j=0}^{r_{k}-1}\left(x^{n_{k}+j+1}-x^{n_{k}+j}\right) \rightarrow x^{*} .
$$

By equalities (38), (39) and by the demi-closedness of $U_{i}-$ Id at 0 , we have $x^{*} \in$ Fix $U_{i}$. Because $i \in L$ is arbitrary, $x^{*} \in \bigcap_{i \in L}$ Fix $U_{i}=C$. We proved that any cluster point of $\left\{x^{k}\right\}_{k=0}^{\infty}$ belongs to $C$. Therefore, the whole sequence $\left\{x^{k}\right\}_{k=0}^{\infty}$ converges weakly to $x^{*}[25$, Theorem 2.16(ii)].

Remark 5.1 Theorem 5.1 differs from a similar result for a more general model [14, Theorems 5.8.15(i) and 5.8.11], where instead of the assumption that $U_{i}$ satisfy the 
DC principle, there is supposed some relation between $U_{i}$ and $P_{\mathrm{Fix} U_{i}}, i \in L$, which should be satisfied for sequences generated by method (34). If $U_{i}$ are nonexpansive, then the weak convergence of sequences $x^{k}$ generated by (34) can be deduced from [25, Theorem 3.20].

Now we consider the SCFPP (6) and the following iteration

$$
x^{k+1}=x^{k}+\lambda_{k}\left(\sum_{i=1}^{p} \nu_{i}^{k}\left(U_{i} x^{k}-x^{k}\right)+\sum_{j=1}^{r} \mu_{j}^{k} \frac{1}{\|A\|^{2}} A^{*}\left(T_{j}\left(A x^{k}\right)-A x^{k}\right)\right)
$$

where $x^{0} \in \mathcal{H}$ is arbitrary, $\lambda_{k} \in[\varepsilon, 1-\varepsilon]$ for some small $\varepsilon>0, v_{i}^{k}, \mu_{j}^{k} \geq 0$ for all $i, \in I, j \in J, k \geq 0$, and $\sum_{i=1}^{p} \nu_{i}^{k}+\sum_{j=1}^{r} \mu_{j}^{k}=1$. One can show that the $C Q$ method of Byrne [2] is a special case of iteration (40) (see Example 6.1). Therefore, we call iteration (40) a block-iterative CQ-type method. Denote $I_{k}=\left\{i \in I: v_{i}^{k}>0\right\}$, $J_{k}=\left\{j \in J: \mu_{j}^{k}>0\right\}, C:=\bigcap_{i \in I} \operatorname{Fix} U_{i}, Q:=\bigcap_{j \in J} \operatorname{Fix} V_{j}$ and $F:=C \cap A^{-1}(Q)$.

Corollary 5.1 Let $U_{i}: \mathcal{H}_{1} \rightarrow \mathcal{H}_{1} V_{j}: \mathcal{H}_{2} \rightarrow \mathcal{H}_{2}$ be QNE, $i \in I, j \in J$, with $F \neq \varnothing, U_{i}$-Id and $V_{j}-\mathrm{Id}$ be demi-closed at $0, i \in I, j \in J$. Let $\left\{x^{k}\right\}_{k=0}^{\infty}$ be generated by iteration (40) with $\nu_{i}^{k} \geq \delta>0$ for all $i \in I_{k}$ and $\mu_{j}^{k} \geq \delta>0$ for all $j \in J_{k}$. If $\left\{I_{k}\right\}_{k=0}^{\infty} \subseteq I$ and $\left\{J_{k}\right\}_{k=0}^{\infty} \subseteq J$ are intermittent, then for an arbitrary starting point $x^{0} \in \mathcal{H}_{1}$, the sequence $x^{k}$ converges weakly to a point $x^{*} \in F$.

Proof Denote $m:=p+r, L:=\{1,2, \ldots, m\}$ and define $U_{i}:=V_{i-p}=$ $\frac{1}{\|A\|^{2}} A^{*}\left(T_{i-p}-\mathrm{Id}\right) A, i=p+1, p+2, \ldots, m$,

$$
\omega_{i}^{k}:= \begin{cases}v_{i}^{k}, & \text { for } i=1,2, \ldots, p \\ \mu_{i-p}^{k}, & \text { for } i=p+1, p+2, \ldots, m\end{cases}
$$

and $L_{k}:=\left\{i \in L: \omega_{i}^{k}>0\right\}$. Note that if $I_{k}$ is $s_{1}$-intermittent and $J$ is $s_{2^{-}}$ intermittent, then $L_{k}$ is $s$-intermittent with $s=\max \left\{s_{1}, s_{2}\right\}$. Because the operators $V_{j}:=\frac{1}{\|A\|^{2}} A^{*}\left(T_{j}-\mathrm{Id}\right) A$ are QNE and satisfy the demi-closedness principle (see Lemma 4.1 with $\alpha=0$ ), the corollary follows from Theorem 5.1.

\section{Examples}

In the examples below we show that in the case $F \neq \emptyset$ the convergence for the methods described in Sect. 1 follows from Corollary 5.1. In the first two examples we consider SFP (8) defined on Hilbert spaces $\mathcal{H}_{1}$ and $\mathcal{H}_{2}$ and suppose that $F \neq \emptyset$.

Example 6.1 Let $U: \mathcal{H}_{1} \rightarrow \mathcal{H}_{1}$ and $T: \mathcal{H}_{2} \rightarrow \mathcal{H}_{2}$ be QNE operators with Fix $U=$ $C, \operatorname{Fix} T=Q$, and satisfying the demi-closedness principle. Consider the following iteration

$$
u^{k+1}=U_{\alpha_{k}}\left(u^{k}+\frac{\beta_{k}}{\|A\|^{2}} A^{*}\left(T\left(A u^{k}\right)-A u^{k}\right)\right),
$$


where $u^{0} \in \mathcal{H}_{1}$ is arbitrary, $\alpha_{k}, \beta_{k} \in[\varepsilon, 1-\varepsilon]$ for some $\left.\varepsilon \in\right] 0,1\left[\right.$, and $U_{\alpha_{k}}$ denotes the $\alpha_{k}$-relaxation of $U$. We can write equivalently $u^{k+1}=U_{\alpha_{k}} V_{\beta_{k}} u^{k}$, where $V_{\beta_{k}}:=$ $\mathrm{Id}+\frac{\beta_{k}}{\|A\|^{2}} A^{*}(V-\mathrm{Id}) A$ is a $\beta_{k}$-relaxation of the operator $V:=\mathrm{Id}+\frac{1}{\|A\|^{2}} A^{*}(T-\mathrm{Id}) A$. Define the sequence $x^{k}$ in the following way:

$$
x^{k+1}= \begin{cases}\left.x^{k}+\frac{\beta_{k}}{\|A\|^{2}} A^{*}\left(T\left(A x^{k}\right)-A x^{k}\right)\right), & \text { if } k \text { is even } \\ U_{\alpha_{k}} x^{k}, & \text { if } k \text { is odd }\end{cases}
$$

where $x^{0}=u^{0}$. Clearly, (42) is a special case of a block-iterative procedure (40) with $p=r=1, U_{1}=U, T_{1}=T$,

$$
\lambda_{k}=\left\{\begin{array}{l}
\beta_{k}, \text { if } k \text { is even, } \\
\alpha_{k}, \text { if } k \text { is odd, }
\end{array} v_{1}^{k}=\left\{\begin{array}{l}
0, \text { if } k \text { is even, } \\
1, \text { if } k \text { is odd, }
\end{array} \text { and } \mu_{1}^{k}=\left\{\begin{array}{l}
1, \text { if } k \text { is even }, \\
0, \text { if } k \text { is odd }
\end{array}\right.\right.\right.
$$

We easily see that $L_{k}$ is 2-intermittent. Because $T$ is QNE and satisfies the DC principle, the operator $V:=\mathrm{Id}+\frac{1}{\|A\|^{2}} A^{*}(T-\mathrm{Id}) A$ is also QNE and satisfies the DC principle (see Lemma 4.1). The weak convergence of $x^{k}$ to a point $x^{*} \in F$ follows now from Corollary 5.1. We have $u^{k}=x^{2 k}$, i.e., $\left\{u^{k}\right\}_{k=0}^{\infty}$ is a subsequence of $\left\{x^{k}\right\}_{k=0}^{\infty}$. This yields the weak convergence of $u^{k}$ to $x^{*}$. Note that iteration (12) is a special case of (41) with $\left.\beta_{k}=\beta \in\right] 0,1$ [. Moreover, iteration (11) is a special case of (41) with $\left.\beta_{k}=\beta \in\right] 0,1\left[, U=2 P_{c}-\mathrm{Id}, T=2 P_{q}-\mathrm{Id}\right.$, where $c: \mathcal{H}_{1} \rightarrow \mathbb{R}$ and $q: \mathcal{H}_{2} \rightarrow \mathbb{R}$ are continuous convex functions which are bounded on bounded subsets. Therefore, the results of Yang [21, Theorem 1], of Moudafi [7, Theorem 2.1] follow from Corollary 5.1. If we set $c(x):=d(x, C)$ and $q(x):=d(x, Q)$, then $\nabla c(x)=x-P_{C} x$ for $x \notin C$ and $\nabla q(y)=y-P_{Q} y$ for $y \notin Q$, and we easily see that iteration (9) reduces to (11), i.e., the result of Byrne [2, Corollary 2.1] in the case $F \neq \emptyset$ also follows from Corollary 5.1 .

Example 6.2 Let $S: \mathcal{H}_{1} \rightarrow \mathcal{H}_{1}$ and $R: \mathcal{H}_{2} \rightarrow \mathcal{H}_{2}$ be $\beta$ - and $\mu$-demi-contractive operators, respectively, where $\alpha, \beta \in[0,1$ [, with Fix $S=C$, Fix $R=Q$, satisfying the demi-closedness principle. Recall that an operator $S: \mathcal{H} \rightarrow \mathcal{H}$ is $\gamma$-demi-contractive, where $\gamma \in[0,1[$, if

$$
\|S x-z\|^{2} \leq\|x-z\|^{2}+\gamma\|S x-x\|^{2}
$$

for all $x \in \mathcal{H}$ and all $z \in$ Fix $S$. Note that $S$ is $\gamma$-demi-contractive if and only if $S$ is a $\frac{1}{1-\gamma}$-relaxation of a QNE operator $U$, i.e.,

$$
S=U_{(1-\gamma)^{-1}}:=\mathrm{Id}+(1-\gamma)^{-1}(U-\mathrm{Id}) .
$$

Moreover, $S_{\alpha}=\left(U_{(1-\gamma)^{-1}}\right)_{\alpha}=U_{\alpha /(1-\gamma)}$. Consider iteration

$$
u^{k+1}=S_{\alpha_{k}}\left(u^{k}+\frac{\beta_{k}}{\|A\|^{2}} A^{*}\left(R\left(A u^{k}\right)-A u^{k}\right)\right)
$$


where $\alpha_{k} \in[\delta, 1-\beta-\delta]$ and $\beta_{k} \in[\delta, 1-\mu-\delta]$ for some small $\delta>0$. If we set $\left.\beta_{k}=\beta \in\right] 0,1[$, then we obtain a method studied in [6] . Iteration (41) can be written as follows:

$$
u^{k+1}=U_{\alpha_{k} /(1-\beta)}\left(u^{k}+\frac{\beta_{k}}{(1-\mu)\|A\|^{2}} A^{*}\left(T\left(A u^{k}\right)-A u^{k}\right)\right)
$$

with $U$ and $T$ being QNE. We easily see that $\alpha_{k} /(1-\beta) \in[\varepsilon, 1-\varepsilon]$ and $\beta_{k} /(1-\mu) \in$ $[\varepsilon, 1-\varepsilon]$ with $\varepsilon:=\min \{\delta /(1-\beta), \delta /(1-\mu)\}>0$. Therefore, method (44) can be reduced to method (41), i.e., [6, Theorem 2.1] follows from Corollary 5.1, similarly as in Example 6.1.

Now we consider SCFPP (6) defined on Hilbert spaces $\mathcal{H}_{1}$ and $\mathcal{H}_{2}$ and suppose that $U_{i}: \mathcal{H}_{1} \rightarrow \mathcal{H}_{1}, T_{j}: \mathcal{H}_{2} \rightarrow \mathcal{H}_{2}$ are cutters satisfying the demi-closedness principle, $i \in I:=\{1,2, \ldots, p\}, j \in J:=\{1,2, \ldots, r\}$, with $F \neq \emptyset$.

Example 6.3 We analyze the convergence behavior of the following method:

$$
u^{k+1}=u^{k}+\gamma_{k}\left(\sum_{i=1}^{p} \alpha_{i}\left(U_{i} u^{k}-u^{k}\right)+\sum_{j=1}^{r} \beta_{j} A^{*}\left(T_{j} A u^{k}-A u^{k}\right)\right)
$$

with $\alpha_{i}>0, \beta_{j}>0, i \in I, j \in J$, and $\gamma_{k}$ satisfying

$$
\varepsilon \leq \gamma_{k} \leq(2-\varepsilon) /\left(\sum_{i=1}^{p} \alpha_{i}+\|A\|^{2} \sum_{j=1}^{r} \beta_{j}\right)
$$

for some small $\varepsilon>0$. This method with constant $\gamma_{k}$ in the case $\mathcal{H}_{1}=\mathbb{R}^{n}$ and $\mathcal{H}_{2}=\mathbb{R}^{m}$ was studied by Censor and Segal in [8]. Its special case with $U_{i}$ and $T_{j}$, $i \in I, j \in J$, being subgradient projections was studied by Censor et al. [10]. Denote

$$
\delta:=\frac{1}{2} \min \left\{\varepsilon \min _{i \in I} \alpha_{i}, \varepsilon \min _{j \in J} \beta_{j}\right\}>0 .
$$

Similarly as in Sect. 5 we easily see that (45) can be written in the form

$$
x^{k+1}=x^{k}+\lambda_{k}\left(\sum_{i=1}^{p} v_{i}^{k}\left(U_{i} x^{k}-x^{k}\right)+\sum_{j=1}^{r} \mu_{j}^{k}\left(V_{j} x^{k}-x^{k}\right)\right)
$$

where $\lambda_{k}=\gamma_{k}\left(\sum_{i=1}^{p} \alpha_{i}+\|A\|^{2} \sum_{j=1}^{r} \beta_{j}\right) \in[\varepsilon, 2-\varepsilon], v_{i}^{k}=\alpha_{i} \gamma_{k} / \lambda_{k} \geq \delta>0, i \in I$, $\mu_{j}=\beta_{j}\|A\|^{2} \gamma_{k} / \lambda_{k} \geq \delta>0, j \in J$, and $\sum_{i=1}^{p} v_{i}+\sum_{j=1}^{r} \mu_{j}=1$. Because $2 U_{i}-$ Id and $2 V_{j}$ - Id are QNE, $i \in I, j \in J$, Corollary 5.1 yields the weak convergence of $x^{k}$ to an element of $F$. Note that this result covers [8, Theorem 4.2] and [10, Theorem 6]. 
Example 6.4 Denote $U:=\sum_{i=1}^{p} \alpha_{i} U_{i}$, where $\alpha_{i}>0, i \in I$, and $\sum_{i=1}^{p} \alpha_{i}=1$, and

$$
V:=\mathrm{Id}+\sum_{j=1}^{r} \frac{\mu_{j}}{\|A\|^{2}}\left(A^{*}\left(T_{j}-\mathrm{Id}\right) A\right)
$$

where $\mu_{j}>0, j \in J$, and $\sum_{j=1}^{r} \mu_{j}=1$. We analyze the convergence of sequences generated by the following three methods:

$$
y^{k+1}=U_{p} V_{\lambda} U_{p-1} V_{\lambda} \ldots U_{1} V_{\lambda} y^{k}
$$

with an arbitrary $y^{0} \in \mathcal{H}_{1}$ and $\left.\lambda \in\right] 0,2[$,

$$
v^{k+1}=U V_{\lambda} v^{k}
$$

with an arbitrary $v^{0} \in \mathcal{H}_{1}$ and $\left.\lambda \in\right] 0,2[$, and

$$
u^{k+1}=U_{i_{k}} V_{\lambda} u^{k}
$$

with an arbitrary $u^{0} \in \mathcal{H}_{1}$, where $i_{k}=k(\bmod p)+1$. Special cases of these methods were studied by $\mathrm{Xu}$ [12], where the operators $U_{i}$ and $T_{j}$ were supposed to be metric projections onto closed convex subsets $C_{i} \subseteq \mathcal{H}_{1}$ and $Q_{j} \subseteq \mathcal{H}_{2}$, respectively, $i \in I$, $j \in J$.

(a) Define a sequence $x^{k}$ by

$$
x^{k+1}= \begin{cases}V_{\lambda} x^{k}, & \text { if } k \text { is even, } \\ U_{i_{k}} x^{k}, & \text { if } k \text { is odd, }\end{cases}
$$

with $x^{0}=u^{0}$. Note that (49) is a special case of a block-iterative procedure (40) with

$$
\begin{aligned}
\lambda_{k} & =\left\{\begin{array}{l}
\lambda, \text { if } k \text { is even, } \\
1, \text { if } k \text { is odd, }
\end{array} v_{i}^{k}=\left\{\begin{array}{l}
0, \text { if } k \text { is even or } i \neq i_{k}, \\
1, \text { if } k \text { is odd and } i=i_{k},
\end{array}\right. \text { and }\right. \\
\mu_{j}^{k} & =\left\{\begin{array}{l}
\mu_{j}, \text { if } k \text { is even, } \\
0, \text { if } k \text { is odd. }
\end{array}\right.
\end{aligned}
$$

Clearly, $I_{k} \subseteq I$ is $(2 p)$-intermittent, and $J_{k} \subseteq J$ is 2 -intermittent. The operator $V$ is a cutter and satisfies the DC principle as a convex combination of cutters satisfying the DC principle (see Theorem 4.1). Therefore, the weak convergence of $x^{k}$ to a point $x^{*} \in F$ follows from Corollary 5.1. For $u^{k}$ given by (48) we have $u^{k}=x^{2 k}$, i.e., $\left\{u^{k}\right\}_{k=0}^{\infty}$ is a subsequence of $\left\{x^{k}\right\}_{k=0}^{\infty}$. Consequently, $u^{k}$ defined by (48) converges weakly to $x^{*}$. Note that this result covers [12, Theorem 3.5].

(b) We easily see that the sequence $\left\{y^{k}\right\}_{k=0}^{\infty}$ defined by (46) is a subsequence of $\left\{u^{k}\right\}_{k=0}^{\infty}$ defined by (48) provided that $y^{0}=u^{0}$. Therefore, $y^{k}$ converges weakly to $x^{*}$. Note that this result covers [12, Theorem 3.3]. 
(c) Define a sequence $x^{k}$ by

$$
x^{k+1}=\left\{\begin{array}{l}
V_{\lambda} x^{k}, \text { if } k \text { is even, } \\
U x^{k}, \text { if } k \text { is odd, }
\end{array}\right.
$$

with $x^{0}=u^{0}$. Note that (50) is a special case of a block-iterative procedure (40) with

$$
\lambda_{k}=\left\{\begin{array}{l}
\lambda, \text { if } k \text { is even, } \\
1, \text { if } k \text { is odd, }
\end{array} v_{i}^{k}=\left\{\begin{array}{l}
0, \text { if } k \text { is even, } \\
\alpha_{i}, \text { if } k \text { is odd, }
\end{array} \text { and } \mu_{j}^{k}= \begin{cases}\mu_{j}, & \text { if } k \text { is even, } \\
0, & \text { if } k \text { is odd }\end{cases}\right.\right.
$$

$i \in I, j \in J$. We easily see that $I_{k} \subseteq I$ and $J_{k} \subseteq J$ are 2 -intermittent. The operators $U$ and $V$ are a cutters and satisfy the DC principle as convex combinations of cutters satisfying the DC principle. Therefore, the weak convergence of $x^{k}$ to a point $x^{*} \in F$ follows from Corollary 5.1. For $v^{k}$ given by (47) we have $v^{k}=x^{2 k}$, i.e., $\left\{v^{k}\right\}_{k=0}^{\infty}$ is a subsequence of $\left\{x^{k}\right\}_{k=0}^{\infty}$. Consequently, $v^{k}$ defined by (47) also converges weakly to $x^{*}$. Note that this result covers [12, Theorem 3.4].

Example 6.5 Consider the following method:

$$
u^{k+1}=U_{i_{k}, \beta_{k}} V_{j_{k}, \alpha_{k}} u^{k},
$$

where $u^{0} \in \mathcal{H}_{1}$ is arbitrary, $\alpha_{k}, \beta_{k} \in[\varepsilon, 2-\varepsilon]$ for some small $\varepsilon>0$ and $\left\{i_{k}\right\}_{k=0}^{\infty} \subseteq I$ and $\left\{j_{k}\right\}_{k=0}^{\infty} \subseteq J$ are almost cyclic controls. Wang and Xu [13] studied a special case of iteration (51) with $p=r, \alpha_{k}=\alpha, \beta_{k}=1$ and $i_{k}=j_{k}=k(\bmod p)+1$. Define a sequence $x^{k}$ by

$$
x^{k+1}=\left\{\begin{array}{l}
V_{j_{k}, \alpha_{k}} x^{k}, \text { if } k \text { is even, } \\
U_{i_{k}, \beta_{k}} x^{k}, \text { if } k \text { is odd, }
\end{array}\right.
$$

with $x^{0}=u^{0}$. Clearly, (52) is a special case of a block-iterative procedure (40) with

$$
\begin{aligned}
& \lambda_{k}=\left\{\begin{array}{ll}
\alpha_{k}, & \text { if } k \text { is even, } \\
\beta_{k}, & \text { if } k \text { is odd, }
\end{array} v_{i}^{k}=\left\{\begin{array}{l}
0, \text { if } k \text { is even or } i \neq i_{k}, \\
1, \text { if } k \text { is odd and } i=i_{k},
\end{array}\right. \text { and }\right. \\
& \mu_{j}^{k}=\left\{\begin{array}{l}
1, \text { if } k \text { is even and } j=j_{k}, \\
0, \text { if } k \text { is odd or } j \neq j_{k} .
\end{array}\right.
\end{aligned}
$$

Obviously, $I_{k}:=\left\{i_{k}\right\} \subseteq L$ and $J_{k}:=\left\{j_{k}\right\} \subseteq J$ are intermittent. Therefore, the weak convergence of $x^{k}$ to a point $x^{*} \in F$ follows from Corollary 5.1. For $u^{k}$ given by (51) we have $u^{k}=x^{2 k}$, i.e., $\left\{u^{k}\right\}_{k=0}^{\infty}$ is a subsequence of $\left\{x^{k}\right\}_{k=0}^{\infty}$. Consequently, $u^{k}$ defined by (51) converges weakly to $x^{*}$. Note that this result covers [13, Theorem 3.2].

\section{Conclusions}

In this paper we proved that a family of strongly quasi-nonexpansive operators having a common fixed point and satisfying the DC principle is closed under convex combinations and compositions (Theorems 4.1 and 4.2). Basing on this fact we proved the 
weak convergence of sequences generated by two block-iterative methods employing strongly quasi-nonexpansive operators satisfying the DC principle (Theorem 5.1 and Corollary 5.1). The first of these methods refers to the common fixed point problem, and the other one refers to the split common fixed point problem. Both results enable a comprehensive study of the convergence behavior of methods for variational inequality problems over a subset of common fixed points of quasi-nonexpansive operators satisfying the demi-closedness principle.

Open Access This article is distributed under the terms of the Creative Commons Attribution License which permits any use, distribution, and reproduction in any medium, provided the original author(s) and the source are credited.

\section{References}

1. Censor, Y., Elfving, T.: A multiprojection algorithm using Bregman projections in a product space. Numer. Algorithms 8, 221-239 (1994)

2. Byrne, C.: Iterative oblique projection onto convex sets and the split feasibility problem. Inverse Probl. 18, 441-453 (2002)

3. Qu, B., Xiu, N.: A note on the CQ algorithm for the split feasibility problem. Inverse Probl. 21, 1655-1665 (2005)

4. Qu, B., Xiu, N.: A new halfspace-relaxation projection method for the split feasibility problem. Lin. Algebra Appl. 428, 1218-1229 (2008)

5. $\mathrm{Xu}, \mathrm{H} .-\mathrm{K}$. .: Iterative methods for the split feasibility problem in infinite-dimensional Hilbert spaces. Inverse Probl. 26, 105018 (2010). 17 pp

6. Moudafi, A.: The split common fixed-point problem for demicontractive mappings. Inverse Probl. 26, 055007 (2010). 6 pp

7. Moudafi, A.: A note on the split common fixed-point problem for quasi-nonexpansive operators. Nonlinear Anal. 74, 4083-4087 (2011)

8. Censor, Y., Segal, A.: The split common fixed point problem for directed operators. J. Convex Anal. 16, 587-600 (2009)

9. Censor, Y., Elfving, T., Kopf, N., Bortfeld, T.: The multiple-sets split feasibility problem and its applications for inverse problems. Inverse Probl. 21, 2071-2084 (2005)

10. Censor, Y., Motova, A., Segal, A.: Perturbed projections and subgradient projections for the multiplesets split feasibility problem. J. Math. Anal. Appl. 327, 1244-1256 (2007)

11. Masad, E., Reich, S.: A note on the multiple-set split convex feasibility problem in Hilbert space. J. Nonlinear Convex Anal. 8, 367-372 (2007)

12. Xu, H.-K.: A variable Krasnosel'skiı̌-Mann algorithm and the multiple-set split feasibility problem. Inverse Probl. 22, 2021-2034 (2006)

13. Wang, F., Xu, H.-K.: Cyclic algorithms for split feasibility problems in Hilbert spaces. Nonlinear Anal. 74, 4105-4111 (2011)

14. Cegielski, A.: Iterative Methods for Fixed Point Problems in Hilbert Spaces. Lecture Notes in Mathematics, vol. 2057. Springer, Heidelberg (2012)

15. Bauschke, H.H., Combettes, P.L.: A weak-to-strong convergence principle for Fejér-monotone methods in Hilbert spaces. Math. Oper. Res. 26, 248-264 (2001)

16. Yamada, I., Ogura, N.: Hybrid steepest descent method for variational inequality problem over the fixed point set of certain quasi-nonexpansive mapping. Numer. Funct. Anal. Optimiz. 25, 619-655 (2004)

17. Cegielski, A.: Generalized relaxations of nonexpansive operators and convex feasibility problems. Contemp. Math. 513, 111-123 (2010)

18. Cegielski, A., Censor, Y.: Opial-type theorems and the common fixed point problem. In: Bauschke, H.H., Burachik, R.S., Combettes, P.L., Elser, V., Luke, D.R., Wolkowicz, H. (eds.) Fixed-Point Algorithms for Inverse Problems in Science and Engineering. Springer Optimization and Its Applications, vol. 49, pp. 155-183. New York, NY, USA (2011) 
19. Opial, Z.: Weak convergence of the sequence of successive approximations for nonexpansive mappings. Bull. Am. Math. Soc. 73, 591-597 (1967)

20. Cegielski, A.: A generalization of the Opial's theorem. Control Cybern. 36, 601-610 (2007)

21. Yang, Q.: The relaxed CQ algorithm solving the split feasibility problem. Inverse Probl. 20, 1261-1266 (2004)

22. Cegielski, A.: Convergence of the projected surrogate constraints method for the linear split feasibility problems. J. Convex Anal. 14, 169-183 (2007)

23. Zhao, J., Yang, Q.: Several solution methods for the split feasibility problem. Inverse Probl. 21, 17911799 (2005)

24. Cegielski, A., Zalas, R.: Properties of a class of approximately shrinking operators and their applications. Fixed Point Theory. 15, 399-426 (2014)

25. Bauschke, H.H., Borwein, J.: On projection algorithms for solving convex feasibility problems. SIAM Rev. 38, 367-426 (1996) 